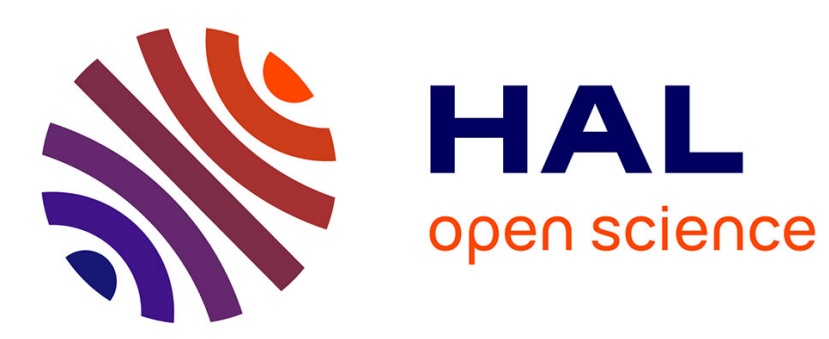

\title{
Tutorat et communauté dans un campus numérique non collaboratif
}

Christine Develotte, François Mangenot

\section{To cite this version:}

Christine Develotte, François Mangenot. Tutorat et communauté dans un campus numérique non collaboratif. Distances et savoirs, 2004, vol. 2 (n²-3/2004), pp.309-333. halshs-00325631

\section{HAL Id: halshs-00325631 https://shs.hal.science/halshs-00325631}

Submitted on 4 Oct 2008

HAL is a multi-disciplinary open access archive for the deposit and dissemination of scientific research documents, whether they are published or not. The documents may come from teaching and research institutions in France or abroad, or from public or private research centers.
L'archive ouverte pluridisciplinaire HAL, est destinée au dépôt et à la diffusion de documents scientifiques de niveau recherche, publiés ou non, émanant des établissements d'enseignement et de recherche français ou étrangers, des laboratoires publics ou privés. 


\title{
Tutorat et communauté dans un campus numérique non collaboratif
}

\author{
Christine Develotte, François Mangenot
}

RÉSUMÉ. Cette étude est fondée sur l'idée qu'entre des approches collaboratives très exigeantes et des environnements à distance sous-estimant l'importance des interactions humaines dans les apprentissages, il existe une place pour des modèles pédagogiques non collaboratifs mais intégrant néanmoins la constitution de liens socio-cognitifs et socio-affectifs, voire la constitution d'une communauté d'apprentissage. L'hypothèse est que les tuteurs jouent un rôle central dans l'établissement de ces liens. La méthodologie adoptée est l'analyse du discours, le corpus étant constitué d'interactions pédagogiques recueillies dans le cadre d'une maîtrise en ligne proposée par le Campus numérique français langue étrangère. Sont plus particulièrement analysées les interventions tutorales et les effets qu'elles suscitent.

ABSTRACT. This paper is based on the assumption that between quite demanding collaborative approaches and distance learning environments which neglect human interactions, there is room for some non-collaborative pedagogical models; even if not aiming at collaboration, these models nevertheless integrate a strong community component. It is assumed that tutors play a central part in the community-building process. Methodology is based on discourse analysis applied to a corpus of pedagogical interactions gathered in a virtual campus aimed at training teachers of French as a foreign language. Tutoral moves and their effects will be analysed in depth.

MOTS-CLÉS : communication médiatisée par ordinateur, apprentissage collaboratif assisté par ordinateur, analyse du discours, tutorat en ligne, communauté d'apprentissage.

KEYWORDS: computer mediated communication, computer supported collaborative learning, discourse analysis, online tutoring, learning community. 
La recherche sur les communautés d'apprentissage en ligne s'est jusqu'à présent surtout intéressée au cas d'environnements visant la collaboration (Henri et al., 2001, Charlier et al., 2003, George et al., 2003, etc.). Dans ces contextes, la collaboration est considérée comme le paradigme d'apprentissage par excellence, en accord avec les théories de la cognition partagée, de la cognition distribuée et de l'apprentissage collaboratif assisté par ordinateur (en anglais CSCL). Un rapide état des lieux des formations en ligne montre cependant que peu d'entres elles s'appuient sur la collaboration, en partie du fait de la lourdeur de ce paradigme exigeant, tant pour les enseignants que pour les apprenants ( $c f$. Arnaud, 2003). Plus encore, c'est souvent la «production et la mise à disposition des ressources numériques » qui prime sur l'accompagnement pédagogique, comme le montre un rapport sur les Campus numériques français (Averous et al., 2002).

Cette étude est fondée sur l'idée qu'entre des approches collaboratives très exigeantes et des environnements à distance sous-estimant l'importance des interactions humaines dans les apprentissages, il existe une place pour des modèles pédagogiques non collaboratifs mais intégrant néanmoins la constitution de liens socio-cognitifs et socio-affectifs, voire la constitution d'une communauté d'apprentissage (expression que nous nous attacherons à définir); on cherchera à vérifier l'hypothèse que les tuteurs jouent un rôle central dans ces l'établissement de ces liens.

La méthodologie adoptée sera celle de l'analyse de discours (Charaudeau et al., 2002), qui nous semble offrir les outils adéquats pour repérer les opérations langagières aptes à susciter des liens entre les participants. Le corpus est constitué d'interactions pédagogiques recueillies à l'intérieur des dix unités d'enseignement d'une maîtrise en ligne proposée par le Campus numérique français langue étrangère (Canufle). Seront plus particulièrement analysées les interventions tutorales et les effets qu'elles suscitent.

Après un tour d'horizon critique de la notion de communauté dans les recherches sur la formation à distance puis une présentation du modèle pédagogique de Canufle, on analysera très précisément les interactions distantes en mettant l'accent sur la constitution du lien social ; on avancera en conclusion quelques suggestions concernant l'analyse de la communication en ligne et la formation des tuteurs

\section{La notion de communauté dans les apprentissages à distance}

On commencera par examiner rapidement comment les sociologues traitent de la notion de communauté virtuelle; les pratiques éducatives s'insèrent en effet dans un contexte sociologique qui n'est pas sans influence sur celles-ci. On examinera ensuite quelques-unes des raisons qui plaident en faveur de l'étayage du lien social à l'intérieur d'un groupe d'apprenants distants. On évoquera enfin diverses typologies ou caractéristiques des communautés en ligne établies par des auteurs du champ des technologies éducatives.

\section{Comment se définit une communauté virtuelle?}

Dans un chapitre intitulé « Les communautés, un autre imaginaire d'Internet », Flichy (2001:85-111) retrace l'historique d'Internet, montrant différentes logiques communautaires qui ont pu s'y développer dans les années 1970 ; il établit un bilan mitigé de « l'utopie communautaire », qui est née du développement des réseaux et qui continue à dominer (surtout aux Etats-Unis) : d'un côté, comme toute utopie, celle-ci a plus promis qu'elle n'a tenu et bien moins nombreux sont ceux qui s'expriment sur Internet que ceux qui observent les débats sans intervenir; de l'autre, cette « idée d'un groupe virtuel où tout le monde s'exprime de façon égalitaire » contribue néanmoins à mobiliser les acteurs. Le même auteur note à propos des Etats-Unis : "Cette société a toujours accordé une grande importance à la notion de communauté. Tocqueville, en son temps, avait déjà noté ce point. »

Est-on alors certain que le terme communauté comporte les mêmes connotations des deux côtés de l'Atlantique et qu'il puisse être traduit littéralement sans autre forme de procès ? La définition de Barry Wellman, citée par Castells (2001: 159), «Les communautés sont des réseaux de liens entre personnes qui apportent de la convivialité, de l'aide, de l'information, un sentiment d'appartenance et une identité sociale. », semble pertinente pour la plupart des communautés en ligne, y compris celles à vocation pédagogique. Elle tient en effet compte de la dimension socio-affective (" convivialité ») autant que cognitive ("information ») et on y pressent l'importance du lien social («appartenance», «identité ») et de l'étayage par les pairs ou par des tuteurs ("aide»). On peut néanmoins se demander quelle traduction française convient le mieux pour les expressions, utilisées dans de nombreux articles sur la formation en ligne (Brown, 2001, Conrad, 2002, par exemple), «community-building» et « sense of community» : les traductions littérales, "constitution d'une 
communauté » et «sentiment communautaire», ne sont-elles pas plus floues qu'une traduction plus interprétative comme « constitution du lien social » et « sentiment d'appartenance à un groupe » ?

Concernant l'adjectif « virtuel », on soulignera, à la suite de Flichy (2001 : 163-164), sa polysémie ou sa « maladresse » (Dillenbourg et al., $2003: 27$ ). Ce terme s'oppose en effet d'une part à « réel », d'autre part à « local» ou «physique» (au sens de présence physique) ${ }^{1}$. Or s'il est clair que certains usages des réseaux, étudiés notamment par Turkle (1995), permettent à des internautes de vivre des expériences bien éloignées de la vie réelle ${ }^{2}$, les pratiques qui intéressent le secteur éducatif se situent plutôt sur le second axe de signification : comme le signalent Dillenbourg et al. (2003), dans communauté virtuelle «l'adjectif ne caractérise pas la communauté mais l'un de ses modes de communication». L'emploi d'expressions comme « communautés délocalisées » (Daele et al., 2003), « cybercommunautés » (Flichy, 2001) ou « communautés en ligne » éviterait alors de laisser penser que ces communautés pourraient avoir un caractère moins réel qu'un regroupement en présence.

Comme on peut le constater, l'expression « communauté virtuelle », surinvestie idéologiquement, prend davantage appui sur l'imaginaire, les pouvoirs fantasmés d'Internet que sur la description précise des spécificités que ce type de communication induit pour les acteurs qui y prennent part. Il s'agirait donc d'essayer de caractériser la nature du lien social qui se tisse dans certains types de communication en ligne et dont on peut, de manière intuitive, reconnaître l'existence. Nous nous proposons donc d'apporter des éléments propres à éclairer l'une de ses principales composantes, à savoir la création de liens sociaux entre les membres d'un groupe communiquant en ligne dans une perspective de formation. La suite de cet article tentera en particulier de répondre à deux questions, à partir de l'analyse des interactions en ligne : le Campus numérique étudié permet-il l'établissement de liens sociaux ? Quel rôle jouent les tuteurs dans l'établissement de ces liens à la base du sentiment d'appartenance à un groupe ?

\section{L'importance du lien social dans les apprentissages à distance}

Le Campus numérique étudié n'ayant pas pour objectif de susciter la collaboration (au sens fort du terme), on ne reprendra pas ici l'argumentaire en faveur d'une construction collective des connaissances. La question du lien social dans les apprentissages à distance sera examinée d'une manière plus générale, indépendante du modèle pédagogique.

De nombreux auteurs abordent le problème de l'abandon (ou du décrochage - terme utilisé au Québec) observé dans l'enseignement à distance. Glickman (2002: 243-254) évoque cinq facteurs-clés : le temps, l'isolement, l'autonomie des apprenants, l'accompagnement et la question du lien social. On remarquera en lisant ce qui suit que ces différents facteurs sont interdépendants.

Quelques rares études de nature qualitative ont enquêté de manière approfondie auprès d'étudiants sur la manière dont ils avaient ressenti le lien social lors d'apprentissages en ligne. On citera Brown (2001), Conrad (2002) et O'Regan (2003), qui ont toutes trois évalué des dispositifs entièrement à distance, selon une même approche ethnographique fondée essentiellement sur de longs entretiens avec les étudiants. La première montre, à partir du cas d'une minorité d'étudiants ( 5 sur 21 ), quels peuvent être les obstacles à l'établissement du lien social : étudiants travaillant essentiellement pour la note et le diplôme, n'imaginant pas a priori qu'une communauté puisse s'établir autrement qu'en face à face, n'arrivant pas à travailler au même rythme que la majorité (toujours en retard), ne consacrant pas suffisamment de temps aux échanges en ligne, soit par empêchement soit par choix personnel. Brown (2001) et Conrad (2002) ont interrogé les étudiants sur leur propre définition du terme « communauté »; dans les deux cas, les étudiants avaient peu de représentations a priori et décrivaient ensuite comme lien social (« community») ce qu'ils avaient vécu dans le cadre de leur cours en ligne. Des deux études se dégage l'impression que les étudiants accordent juste ce qu'il faut d'interactions au lien social pour travailler dans une ambiance agréable (ils semblent conscients de cette nécessité), mais qu'ils ne sont pas prêts à consacrer une part plus grande de leur temps - toujours compté - à cette dimension ; des différences individuelles assez fortes sont cependant relevées, certains appréciant les échanges à caractère purement phatique (comme les blagues). O'Regan, pour sa part, a étudié les émotions, négatives et positives, des étudiants en ligne : frustration devant une technologie qui ne marche pas toujours, devant un contenu en ligne mal structuré, anxiété face au temps à gérer, face à des consignes de travail peu explicites, face au regard des

1. Flichy étudie également une troisième acception, celle que prend le terme dans le syntagme réalité virtuelle. Il s'agit alors de simulation, mais cela n'a plus rien de social.

2. L'expression « real life », opposée aux « virtual worlds », en témoigne notamment. 
pairs inconnus, face aux feed-back tutoraux qui tardent ou ne viennent pas, embarras ou honte liés au caractère public et permanent des écrits publiés, enthousiasme devant la nouveauté de ce mode d'apprentissage, devant les possibilités offertes par la technologie, fierté quand on a l'impression d'avoir bien réussi une tâche et que l'on reçoit un feed-back positif de l'enseignant et des pairs. L'auteur conclut d'une part en donnant un certain nombre de conseils pratiques destinés à éviter les émotions négatives, d'autre part en affirmant que la question des émotions ne doit pas être écartée de la recherche sur les environnements en ligne. Comme on l'a indiqué précédemment, les auteurs cités ci-dessus s'appuient sur des témoignages d'étudiants plutôt que sur leurs pratiques réelles. En complémentarité avec leurs études, nous avons choisi, ici, de fonder notre recherche sur l'analyse des interactions en ligne. Il pourra être intéressant d'examiner si un autre type de données révèle d'autres dimensions du lien social et quels types de rapprochements peuvent être établis.

\section{Caractéristiques des communautés en ligne}

Différents auteurs du champ des technologies éducatives ont analysé les communautés en ligne, tantôt en fonction de la force du lien qui unit les membres, tantôt en fonction de leur finalité. Pour Henri et al. (2002: 28), il existe quatre formes de communautés virtuelles, la communauté d'intérêt, la communauté d'intérêt intelligente, la communauté d'apprenants et la communauté de pratique :

Ces communautés partagent des caractéristiques communes, mais à des degrés différents. De la communauté d'intérêt à la communauté de pratique, l'engagement, la participation, l'entraide, le partage de significations communes et l'affirmation de l'identité commune gagnent en intensité au fur et à mesure que s'affirme l'intention de réaliser le projet communautaire et que se resserrent les liens entre les membres de la communauté. Ainsi, le projet d'une communauté peut se développer et se préciser au fil des interactions des participants.

La classification proposée par ces auteurs constitue plus un instrument de mesure de certaines dimensions (lien social, collaboration) qu'une typologie permettant de caractériser telle ou telle communauté. La gradation du lien social proposée est par ailleurs discutable : pourquoi celui-ci serait-il moins fort dans une « communauté d'intérêt intelligente», comme celle des patients atteints d'une maladie évolutive, que dans une " communauté de pratique », composée par exemple d'enseignants mutualisant leurs idées pédagogiques ?

Se défendant de toute intention typologique, Dillenbourg et al. (2003) utilisent trois catégories identiques à celles de Henri et al., mais ils mettent l'accent, dans les exemples qu'ils fournissent, sur l'objectif des groupes constitués plutôt que sur le lien qui les unit. Ainsi, les communautés d'intérêt regroupent-elles des individus qui partagent une préoccupation commune, par exemple une maladie chronique. Les communautés de pratique réunissent des membres d'une même profession cherchant à mutualiser une certaine expertise. Les communautés d'apprentissage sont des groupes de personnes se rassemblant pour acquérir des connaissances. On distingue ainsi trois grandes familles d'objectifs, pas forcément exclusifs les uns des autres : l'entraide, la mutualisation et l'apprentissage. Ces mêmes auteurs préconisent « une utilisation parcimonieuse du terme de communauté » dans le domaine des apprentissages en ligne ; selon eux, le déterminisme technologique doit être évité et un certain nombre de conditions remplies pour que le terme puisse être utilisé :

Un groupe d'étudiants qui interagit par courriel ou au sein d'un forum ne constitue pas automatiquement une communauté au sens strict du terme. Un environnement informatique ne crée pas une communauté virtuelle si ses membres ne sont pas interdépendants, ne partagent pas une microculture, ne s'insèrent pas dans une structure organique et si l'espace ne leur appartient pas.

Enfin, certains auteurs observent l'évolution des communautés virtuelles dans le temps. Brown distingue trois phases correspondant à un engagement de plus en plus important, une première de prise de contact (« making on-line acquaintances »), une seconde caractérisée par des interactions plus construites et plus signifiantes ( " community conferment»), une dernière qu' elle nomme " camaraderie » (sic) et qui se caractérise par l'intensité des échanges; certains étudiants ne dépassent pas la première ou la seconde phase. C'est également une évolution en trois phases que proposent Dillenbourg et al., tout en mettant, quant à eux, davantage l'accent sur la dimension cognitive : co-construction progressive des références, puis d'un jeu de concepts, enfin, à plus long terme, d'une culture.

\section{Synthèse}

Pour resituer notre étude par rapport aux travaux évoqués dans cette partie, précisons que la communication inter-individuelle que nous allons observer correspond clairement, en termes de finalités, à ce que les auteurs 
cités appellent " communauté d'apprentissage » : les étudiants fréquentent Canufle avant tout afin d'obtenir un diplôme et d'acquérir des connaissances nouvelles. Une dimension de confrontation et de mutualisation des pratiques pédagogiques n'est cependant pas absente.

Notre objectif étant de préciser le type de relations qu'entretiennent les acteurs les uns avec les autres via Internet, le terme " communauté » présente-t-il alors un intérêt épistémologique ? On a vu que certains auteurs insistaient tantôt sur la dimension socio-affective, tantôt sur les aspects cognitifs ou socio-cognitifs, tandis que d'autres se focalisaient plus sur la collaboration ou sur le sentiment d'appartenance au groupe ou bien encore sur les finalités des acteurs. Le terme "communauté » présente alors sans doute l'avantage d'entremêler ces différentes dimensions difficiles à distinguer dans la réalité, mais il ne doit pas empêcher les chercheurs d'étudier tel ou tel aspect de manière plus fine. Pour notre part, ce sont les dimensions socio-affective et socio-cognitive, ainsi que le sentiment d'appartenance au groupe qui retiendront notre attention. Après avoir précisé dans la partie suivante la structure communicative du Campus numérique, nous partirons du point de départ de la naissance du groupe, pour analyser l'établissement des liens sociaux entre les différents acteurs (tuteurs et apprenants) au cours des deux premiers mois de formation.

\section{Le « modèle pédagogique » Campus numérique FLE (Canufle)}

Un possible malentendu doit tout d'abord être levé : l'emploi du terme «modèle » n'est pas lié à l'idée d'objet à imiter, mais bien plutôt à la notion de « représentation simplifiée d'un processus, d'un système " (Petit Robert 1). A l'heure où de nombreuses instances s'intéressent à la modélisation des «objets pédagogiques " utilisés dans la formation ouverte et à distance, la description précise d'un dispositif et de ses variables, bien que se situant à un niveau plus " macro ", s'inscrit dans cet effort de description. Le modèle pédagogique de Canufle a déjà été décrit assez précisément dans deux articles (Mangenot, 2002 et 2003), aussi se contentera-t-on ici, après une brève présentation de sa genèse, d'en décrire quelques caractéristiques liées à la problématique de cet article : le modèle communicationnel, la qualité et le rôle des tuteurs.

\section{Genèse de Canufle}

Les premières expérimentations remontent à l'année 1999-2000. L'université Grenoble 3, en partenariat avec le CNED, proposait alors une maîtrise de français langue étrangère à distance comportant plus de 500 inscrits et entièrement fondée sur le support papier ; le seul suivi consistait en la correction d'un devoir d'entraînement par unité d'enseignement (UE) et par an. L'initiative a alors été prise de proposer un suivi en ligne pour une des UE, un module optionnel de $50 \mathrm{~h}$ intitulé Technologies de l'information et de la communication et enseignementapprentissage du FLE (Mangenot, 1999). Les étudiants ayant choisi cette option, au nombre d'une centaine, avaient été prévenus qu'un accès à Internet était requis. Le cours, sous forme d'une brochure de 170 pages, avait été rédigé dans la perspective d'un suivi en ligne et chacun des huit chapitres se terminait par un certain nombre de tâches ouvertes. Chaque étudiant, de manière individuelle, devait «publier » régulièrement (à raison d'un chapitre toutes les trois semaines) ses travaux sur un système de type forum, le suivi étant assuré par l'auteur du cours. La participation a été relativement importante et régulière et un questionnaire envoyé aux étudiants en fin d'année a montré un taux de satisfaction élevé, même de la part d'étudiants qui avaient très peu contribué. La charge de travail pour l'enseignant-tuteur est restée dans des proportions tout à fait raisonnables (de l'ordre de 3 à $4 \mathrm{~h}$ par semaine durant six mois) et a pu être rémunérée grâce à une subvention de Grenoble Campus Ouvert $(\text { Greco })^{3}$.

En 2000-2001, deux autres enseignants ont suivi la même voie ; il s'agissait cette fois d'UE obligatoires, n'ayant rien à voir avec les TIC. La participation au suivi par Internet avait un caractère facultatif. Dans l'un des deux cas, celui d'un enseignant ayant proposé des tâches précises et assuré un suivi régulier, l'expérience s'est révélée concluante; dans l'autre cas, l'enseignante avait simplement prévu un tutorat réactif sans proposer de tâches et la participation étudiante est restée très limitée.

C'est à la faveur du second appel d'offre «Campus numériques » du Ministère de l'éducation français que l'idée de généraliser ces premières expériences à l'ensemble de la maîtrise FLE (350h de cours) a pris corps. L'appel d'offre impliquait un partenariat entre plusieurs universités et le travail supplémentaire entraîné par le suivi en ligne d'étudiants n'aurait pas trouvé suffisamment de volontaires parmi les enseignants de Grenoble 3.

3. Organisme interuniversitaire d'aide à l'intégration des TICE et au développement de la FOAD (http://greco.grenet.fr). 
L'université Lyon 2 et l'ENS Lettres et sciences humaines (Lyon) dans un premier temps (toujours en partenariat avec le CNED), puis l'université de Bourgogne et celle de Franche-Comté dans un second temps, se sont donc associées au projet, qui a été retenu et subventionné par le Ministère. En 2002-2003, 175h de cours (quatre UE) ont ainsi été proposées selon les modalités indiquées plus haut, à ceci près que les cours n'étaient plus sous forme de brochures envoyées par la poste mais téléchargeables sous format PDF. En 2003-2004, c'est la maîtrise de FLE complète qui est proposée dans une version « en ligne », parallèlement à la version traditionnelle, pour un coût environ deux fois supérieur, justifié par le meilleur suivi.

\section{Le modèle communicationnel de Canufle}

Comme cela a déjà été indiqué, Canufle ne fonctionne pas selon un modèle collaboratif, à l'opposé d'autres dispositifs s'adressant à des publics similaires, comme Learn-Nett (cf. Charlier et al., 2003). Les raisons de ce choix sont multiples, mais elles sont pour la plupart liées à la lourdeur des dispositifs faisant appel à la collaboration dès que l'on a affaire à des groupes importants d'étudiants. Tout d'abord, la collaboration implique la constitution puis le bon fonctionnement de groupes de taille modeste : avec des étudiants n'ayant jamais la possibilité de se rencontrer, d'importants problèmes à la fois logistiques et psychopédagogiques doivent être à surmontés. Ensuite, un travail collaboratif présuppose que l'on ait déterminé précisément un produit à réaliser : toutes les UE d'une maîtrise universitaire ne se prêtent pas forcément à ce type de production. Enfin, chaque groupe doit être suivi par un tuteur, ce qui implique soit que chaque tuteur s'occupe de plusieurs groupes, soit qu'on dispose d'un grand nombre de tuteurs.

Quel est alors le modèle communicationnel de Canufle ? On peut le résumer en trois points : tout d'abord le fait que les contributions des étudiants soient sollicitées par des tâches mises en ligne mensuellement, ensuite le suivi hebdomadaire par les enseignants-tuteurs ${ }^{4}$ des contributions des étudiants en réponse aux tâches, enfin le caractère écrit, asynchrone et " public » (" many to many ») de toutes les interactions.

L'approche fondée sur la tâche adoptée dès les premières expérimentations trouve son origine chez certains didacticiens des langues anglo-saxons, qui prônent depuis longtemps une telle approche dans l'apprentissage des langues étrangères. Elle a depuis été confortée par des spécialistes de la formation en ligne, comme Oliver $e t$ al. (2001). Les contacts, à travers Greco, avec d'autres collègues ayant expérimenté des suivis en ligne avaient également montré que si l'on se contentait d'attendre les sollicitations des étudiants pour y réagir (tutorat réactif), on observait généralement une participation très limitée de leur part.

Le caractère écrit et asynchrone des interactions relevait, dans notre cas, de l'évidence, étant donnée la dispersion des étudiants sur tous les continents. L'idée de rendre toutes les interactions publiques se fondait pour sa part sur plusieurs prémisses. Tout d'abord, on souhaitait éviter le sentiment d'isolement (cf. supra). Une communication bilatérale étudiant-tuteur par courriel vient certes rompre partiellement cet isolement, mais pas au même degré que si l'on donne la possibilité aux étudiants de se lire les uns des autres et de communiquer entre eux. Ensuite, sachant que l'on avait le plus souvent affaire à des étudiants déjà engagés dans l'enseignement, on visait une certaine mutualisation des expériences. Au-delà de cette mise en commun, on pouvait également espérer des confrontations d'opinions génératrices de conflits socio-cognitifs, à l'instar de ce qui peut se produire lors de travaux dirigés présentiels ; mais on était bien conscient que cette dernière dimension était en grande partie dépendante de la nature des tâches. Un dernier avantage du caractère public des échanges est le gain de temps que cela entraîne, l'enseignant n'ayant pas à répondre plusieurs fois aux mêmes questions.

\section{Qualité et rôle des tuteurs}

Le tutorat à distance correspond à un certain nombre de fonctions, pas forcément remplies par les mêmes personnes et pas toujours assurées dans chaque dispositif, qui ont été répertoriées par différents auteurs (Deschryver, 2003, Denis, 2003). L'importance relative de ces fonctions dépend du modèle pédagogique : un tuteur dans un projet collaboratif, par exemple, remplira certaines fonctions spécifiques à la collaboration ( $c f$. Deschryver, 2003). Dans le cas de Canufle, les types d'interventions tutorales proposées par Denis (2003) semblent pertinentes, à l'exception de l'accompagnement technique, assuré pour l'ensemble du dispositif par un

4. Il s'agit là du terme retenu dans la convention cadre du consortium Canufle; l'ensemble tâches/suivi est par ailleurs nommé « travaux dirigés en ligne », afin de rester au plus près des dénominations dans le cas du présentiel, ce qui facilite les discussions avec les conseils d'administration sur la rémunération de cette nouvelle forme d'enseignement. 
ingénieur d'études : accueil et mise en route, accompagnement disciplinaire, accompagnement méthodologique, auto-régulation et méta-cognition, évaluation, rôle de personne-ressource attitrée. Selon Denis, ces six fonctions demandent des compétences disciplinaires, pédagogiques et relationnelles.

Dans le cadre du modèle Canufle, on souhaitait tout d'abord que les tuteurs possèdent des compétences disciplinaires : cela permet d'une part au tuteur de ne pas à avoir à dépendre d'un spécialiste (ce qui complexifierait le dispositif), cela renforce par ailleurs la crédibilité du tuteur aux yeux des étudiants. Un des premiers choix a donc consisté à ne faire appel qu'à des collègues enseignant déjà dans le même diplôme en présentiel. Mais une nouveauté importante a tout de même été introduite : chaque UE est encadrée par deux collègues, appartenant à deux universités différentes. Au départ, l'un de ces deux collègues était généralement aussi le rédacteur du cours, l'autre étant un spécialiste du même domaine ou d'un domaine proche.

Pour les compétences pédagogiques, les savoir-faire en face à face ne sont pas forcément transférables à la $\mathrm{FAD}$; mais on pensait que l'approche par tâches pourrait être assez facilement appropriée par les collègues. A ce sujet, il convient de signaler que la conception des tâches d'apprentissage incombe habituellement à l'auteur du cours; aucun des auteurs cités plus haut ne considère qu'il puisse s'agir d'une fonction tutorale. Or dans Canufle, ce sont les tuteurs qui conçoivent les tâches, suivant en cela les conseils de Oliver et al. (2001), pour qui ressources et tâches gagnent à être dissociées afin de permettre une meilleure réutilisation des ressources ; un cours peut ainsi avoir une durée de vie assez longue sans que la pédagogie en soit figée, puisqu'il est possible d'ajuster les tâches chaque année. Concernant le cadrage temporel, fonction pédagogique importante dans la $\mathrm{FAD}$, les enseignants-tuteurs n'ont pas à s'en préoccuper : il est assuré par le dispositif global qui prévoit un rythme identique pour toutes les UE, à savoir une mise en ligne mensuelle (de novembre à avril) de nouvelles tâches correspondant à une nouvelle partie du cours et un délai d'un mois pour réaliser ces tâches.

Pour ce qui est des compétences relationnelles, enfin, on n'avait pas une idée précise au départ de la manière dont celles-ci peuvent se décliner dans une situation entièrement à distance... Ce sera justement l'objet de cette étude que d'essayer de mieux caractériser certaines de ces compétences. Mais on était à la recherche d'un modèle relationnel qui assure suffisamment de présence auprès des apprenants ${ }^{5}$ et en même temps ne soit pas trop coûteux en temps : l'engagement pris par les tuteurs est celui d'une connexion hebdomadaire au minimum, ce qui s'est révélé suffisant. On estimait également que face à un mode de communication totalement nouveau, travailler en binôme serait à la fois plus rassurant et plus enrichissant pour les tuteurs; ce système permettait par ailleurs aux étudiants d'être en contact avec deux manières différentes d'aborder les contenus et positionnait les différents acteurs au sein d'équipes : celle des enseignants-tuteurs et celle des étudiants.

\section{Analyse discursive du lien social}

Le corpus sur lequel s'appuie cette étude est composé des 466 contributions qui ont été placées sur la plateforme du campus numérique par les tuteurs et les étudiants de maîtrise FLE lors des deux premiers mois de formation (182 contributions de tuteurs, 284 d'étudiants). Le cursus se subdivise pour 2003-2004 en dix UE différentes. Pour étudier la façon selon laquelle se construit discursivement un sentiment de communauté à partir du dispositif énonciatif proposé par le campus numérique, nous chercherons à repérer dans le discours des tuteurs, à partir des outils classiques de l'analyse du discours (Charaudeau et al., 2002), les incitations à créer un lien socio-cognitif entre les étudiants et socio-affectif entre eux et les étudiants. Cette première étape nous permettra de mettre au jour ce qui relève de l'ordre du prescrit dans l'établissement des liens sociaux entre les acteurs du campus numérique. Dans un deuxième temps, nous chercherons à voir l'impact de ces incitations discursives à travers les interactions pédagogiques et plus précisément à partir de l'analyse de plusieurs «points d'accroche » interactionnels, tirés d'UE différentes. La focalisation sur des lieux discursifs précis nous fournira des éléments concernant la nature du lien social qui s'exprime dans l'ordre du réel.

\footnotetext{
5. Extrait de la convention cadre Canufle à propos du tutorat numérique : « Ce service constitue la raison d'être du dispositif Canufle. Il s'agit d'un accompagnement pédagogique fort, qui permet de lutter efficacement contre le sentiment d'isolement auquel sont soumis les étudiants relevant des dispositifs de FOAD. »
} 


\section{Du prescrit : l'incitation des tuteurs à créer du lien social}

\section{Les pages de présentation des activités mensuelles}

Nous nous concentrerons, dans cette étude, sur les espaces discursifs directement destinés aux interactions pédagogiques, signalant simplement qu'existent des espaces de présentation personnelle (enseignants et étudiants) et de discussion informelle. Un premier espace discursif est constitué par les écrans de présentation des activités mensuelles, écrans apparaissant dès que l'étudiant clique sur le mois en cours et énonçant les consignes de travail pour ce mois. Nous prélèverons donc, pour notre analyse, des discours issus de ces écrans d'accueil et nous poursuivrons leur étude au paragraphe suivant à partir du type de tâche qu'ils proposent aux étudiants.

Trois exemples permettent d'illustrer les différentes formes que peuvent prendre les incitations à constituer une culture de groupe. Ils sont tirés des UE indiquées ci-dessous :

\section{Sociologie du langage}

Nous attirons votre attention sur l'utilité de lire les contributions de vos collègues ainsi que nos commentaires qui s'y réfèrent : il n'est pas interdit de répondre aux réflexions des uns et des autres (celles des tuteurs y compris), que ce soit pour demander des précisions, en apporter à votre tour ou seulement bénéficier de la richesse d'autres expériences. On peut évoquer la valeur formative de cet échange, car l'investissement dans les réflexions des collègues peut vous permettre d'observer certaines erreurs et de ne pas les reproduire lorsque vous rédigez votre devoir.

\section{Lettres et arts contemporains}

Novembre, tuteur 2 : Découvrir, travailler autrement, échanger, jouer, tels sont les mots clefs qui devraient nous guider dans nos rendez-vous mensuels. Sans omettre le plaisir.

Décembre, tuteur 1 : novembre vous a permis de vous interroger avec bonheur sur vos musées et galeries imaginaires et vos dix romans pour île déserte. Si vous ne l'avez pas encore fait, allez consulter (il est encore temps) les contributions des unes et des autres.

\section{Linguistique textuelle}

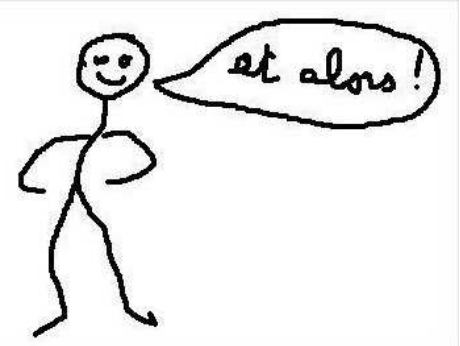

Je suis bien placé pour savoir que nous n'avons pas réussi notre rentrée universitaire canufle 2003-2004 comme nous l'avions souhaité. Concrètement, nous sommes pour cette année encore avec un nombre (trop) restreint d'inscrits pour favoriser une véritable dynamique de groupe, notamment pour les enseignements déjà ouverts en 2002-2003 et déjà suivis "brillamment" par la première promotion Canufle.

Mais cette promotion a maintenant investi les plates-formes correspondant aux enseignements ouverts en 2003-2004. Et, dommage collatéral... les "vieux" ont entraîné, ont "aspiré" les jeunes qui ont tendance à privilégier eux-mêmes ces enseignements..

C'est pô juste!

Ne me laissez pas sombrer dans la neurasthénie en décembre!

Si le premier exemple explicite, sous la forme argumentative classique du discours prescriptif pédagogique, l'utilité de s'intéresser aux productions des autres étudiants en les lisant, voire en y répondant, donc en incitant à la coopération, le second, par l'emploi du champ sémantique du plaisir, trouve un autre ressort argumentatif à la même injonction finale : s'intéresser à ce que font les autres. Par ailleurs, l'emploi de l'adjectif possessif à la première plutôt qu'à la deuxième personne dans « nos rendez-vous mensuels » est également propre à créer une impression de motivation réciproque étudiants-tuteurs dans les rencontres régulières sur le campus ${ }^{6}$. Quant au troisième, jouant sur la multicanalité offerte par le support en ligne, il met en scène un petit bonhomme, mirigolard mi-fouettard, censé mettre au travail de façon humoristique les étudiants qui tarderaient trop à le faire. Le fait que ce cours soit dirigé par un seul tuteur confère une implication plus personnelle au « je » qui s'exprime et qui joue sur un registre d'expression enfantin, tant au niveau du dessin que du mime de l'oralité par l'écriture

6. On a pu ainsi mettre en évidence (Develotte, 2004) le fait que ce genre de communication pédagogique en ligne a tendance à privilégier l'emploi des pronoms personnels pluriels : d'une part les enseignants-tuteurs s'adressant à différents étudiants en même temps (et même au groupe-classe en tant que surdestinataire), d'autre part l'étudiant s'adressant aux tuteurs du module. 
« C'est pô juste! ! ${ }^{7}$ pour tenter, par le ton d'enfant boudeur et dépité dont il use, d'attendrir les étudiants et de les attirer vers son cours. La forme de supplication que revêt la phrase finale marque d'ailleurs un renversement des rôles, le tuteur, dans son besoin de dialogue, se plaçant en position de demande d'assistance à ses étudiants.

\section{Les tâches demandées}

Ces tâches sont souvent sous-tendues par l'intention de la part du tuteur de créer des liens entre les étudiants. La majorité des UE cherche en effet, dans les tâches proposées, à impliquer personnellement les étudiants par le biais de questions qui les amènent à se décrire, s'auto-analyser, en bref à produire un discours subjectif, personnel. Nous en analyserons six exemples :

\section{a) Evolution méthodologique et apprentissage du FLE}

Pour novembre,

- je vous demanderai tout d'abord de tracer votre profil en matière de FLE ou plus généralement de langue vivante étrangère : quelle est votre discipline d'origine ? êtes-vous enseignant ? [...]

- je vous demanderai de vous pencher sur votre propre expérience d'apprenant de langue étrangère. Essayez de décrire le plus concrètement possible à travers quelles pratiques vous avez étudié les LVE.

- gardez-vous un bon souvenir de certains de vos apprentissages de langue ? ou au contraire un souvenir peu favorable? Dans les deux cas, essayez de décrire concrètement à quoi est due cette impression favorable ou défavorable ?

\section{b) Sociologie du langage (novembre)}

D'après votre propre expérience d'apprentissage et d'utilisation de différentes langues, ou en fonction des observations que vous avez pu faire dans votre environnement, quelles fonctions attribuez-vous au "parler bilingue » et quelles images et représentations sociales vous semblent généralement associées à ces phénomènes et à ces pratiques ?

c) Approches interculturelles (décembre)

- Quels sont les stéréotypes de la langue française et des Français qui circulent dans le pays où vous vous trouvez. Comment les expliquez vous?

- Quelles sont les représentations des langues étrangères enseignées dans le pays où vous vous trouvez ? (faites un sondage auprès de vos étudiants ou de votre entourage)

d) TICE et FLE (novembre)

Pensez-vous que vous écrivez différemment avec le traitement de texte et sur papier ? Expliquez.

\section{e) Stage-Modalités d'observation de classes}

Pour ceux qui n'ont jamais enseigné le FLE, je vous propose de m'envoyer comme premier travail vos représentations actuelles sur le métier d'enseignant de fle.

\section{f) Grammaire de l'oral}

- Novembre: Je vous propose un peu d'auto-observation sur l'importance relative de l'oral et de l'écrit dans notre quotidien. Pensez à la journée d'hier et essayez d'inventorier ce que vous avez dit à quelle occasion, les conversations que vous avez pu avoir, dans quelles circonstances et avec quels interlocuteurs, dans quelles situations d'écoute pure vous êtes-vous trouvé(e), qu'avez-vous eu l'occasion de lire en précisant le type d'écrit? Vous est-il arrivé d'écrire d'une façon ou d'une autre (manuelle ou sur clavier), quels types d'écrits ? [...] J'aimerais par la suite travailler sur un corpus oral avec toute la classe. [...] Je demande aux résidents de pays francophones d'enregistrer une ou deux conversations supplémentaires pour éventuellement dépanner quelqu'un de très isolé.

- Décembre : J'aimerais que nous constituions ensemble un corpus de transcription d'enregistrements de conversations téléphoniques radiophoniques.

Ces six matières font ainsi appel aux représentations des étudiants ( $c f . \mathrm{d}$, e), à l'observation de leur environnement local/géographique ou temporel/historique ( $c f$. c), d'eux-mêmes ( $c f$. a, f) voire à leur subjectivité ( " gardez-vous un bon souvenir/ peu favorable? », a). Par ailleurs le recueil de données tel que celui induit par l'activité f permet de collecter des informations sur le quotidien des étudiants mêlant intimement vie privée/vie consacrée à Canufle, comme on le vérifiera dans la partie qui suit. Ces différentes entrées dans la formation sont autant d'occasions de présentation de soi à travers le prisme thématique d'une tâche pédagogique. Si les tuteurs ne s'intéressent, en principe, qu'aux réponses apportées aux questions qu'ils ont posées et n'ont accès qu'à une des facettes de présentation des étudiants, en revanche, les étudiants doivent finir par se connaître relativement mieux qu'ils n'auraient pu le faire en présentiel, en tout cas si l'on en juge par la somme d'information sur les autres étudiants qu'ils ont été amenés à glaner au fil des modules, à la lecture des contributions de leur collègues.

La deuxième partie de l'exemple $\mathrm{f}$ met de plus en évidence un type de collaboration au cours de laquelle tuteur et étudiants mettront la main à la pâte pour " constituer ensemble » un corpus spécifique. Non seulement le fait de s'associer au projet mais également de concevoir les différents apports comme contribuant à un projet 
unique est de nature à fournir un élément de cohésion pour le groupe-classe. D'autant plus que le tuteur prévoit également l'organisation d'une procédure d'entraide entre étudiants (" pour éventuellement dépanner quelqu'un de très isolé »). L'ambiance suscitée dans le groupe par cette précaution méthodologique prise par le tuteur est également susceptible d'imprégner l'ensemble de la formation, dans une formule de travail plus coopérative que compétitive.

\section{Au réel : Les interactions tuteurs-étudiants et étudiants entre eux}

\section{Les réponses faites aux contributions des étudiants}

L'espace discursif où «se rencontrent» étudiants et tuteurs est constitué par les forums où les étudiants viennent placer les contributions répondant à telle ou telle tâche. Nous nous intéresserons ici aux contributions réactives des tuteurs, c'est à dire à celles qui viennent en réponse à des productions discursives d'un ou de plusieurs étudiants. Nous chercherons alors à identifier, qualitativement, la nature des liens socio-cognitifs et socio-affectifs exprimés. Les exemples qui suivent sont extraits de réponses effectuées soit à une contribution d'un étudiant $(\mathrm{a}, \mathrm{b}, \mathrm{cl})$ soit aux contributions d'étudiants dans leur globalité en réponse collective au groupe (c2).

a) Lettres et arts contemporains

Je vous avoue que ces photographes m'étaient inconnus et que la visite des sites que vous proposez est passionnante. J'encourage donc vos camardes à les visiter. Canufle, c'est aussi fait pour cela.

b) Linguistique textuelle

Fragments ou extraits ? Autant j'approuve la très grande majorité de vos remarques (cf. ma réponse à S.), autant j'émets des réserves quant à votre audace finale. [...]

c) Grammaire de l'oral

- c1) : Merci V, pour ce travail tout à fait satisfaisant! J'encourage vos camarades à en prendre connaissance. (17/12)

- c2) : J'ai lu avec beaucoup d'intérêt vos différentes contributions sur la «journée d'hier », elles m'ont permis de vous découvrir un peu dans vos individualités respectives. Ce n'était pas prémédité, mais le fait de vous faire analyser une journée de votre vie m'a fourni des impressions beaucoup plus « vivantes » que ne l'aurait fait le rituel questionnaire de renseignements rempli en début d'année par les étudiants présents dans nos classes. J'ai découvert votre éparpillement géographique, vos contraintes professionnelles, familiales, etc. Heureusement, beaucoup d'entre vous ont enfreint la règle des 150 mots et ont ainsi pu fournir des détails très réalistes! Si vous ne l'avez pas encore fait, prenez le temps de lire les contributions de vos petits camarades de classe : vous apprendrez à mieux vous connaître. En tout cas, merci pour ces tranches de vie! (19/12)

Dans le premier exemple, le fait que le tuteur commence par reconnaître son ignorance préalable (« je vous avoue ») est en soi intéressant car on voit se rejouer l'inversion précédemment pointée entre professeur (savant) et étudiant (ignorant). L'emploi du verbe " avouer» laisse à penser que le tuteur envisage cette ignorance comme une lacune de sa part (on avoue des torts ou une faute) et qu'il assume pleinement la prise de risque qui va de pair avec le fait de «donner prise sur lui» (comment? Le tuteur ne connaît pas cela ?) (KerbratOrecchioni, 1997 : 111). Ce que l'on cherche à suggérer ici, c'est que le système des faces étudiées dans les situations de face à face (Goffman) ne se gère peut-être pas à l'identique sur la scène numérique. Le deuxième exemple donne une occurrence d'intertextualité, en mentionnant dans la réponse qui est faite une autre réponse faite ailleurs à un autre étudiant. Comme on le verra, ce procédé de renvoi d'un discours à un autre est repris par les étudiants, les comportements discursifs étant bien entendu susceptibles d'être reproduits, a fortiori s'ils proviennent du tuteur.

Du dernier exemple nous retiendrons tout d'abord une certaine spontanéité dans la transmission de l'intéêt pris à la lecture, "ce n'était pas prémédité ", laissant entendre que la surprise des réponses est de l'ordre des surprises de la vie. D'ailleurs le fait d'employer des lexèmes tels que " vivantes », « réalistes », ou l'expression «tranches de vie» tente bien d'inscrire les «impressions» et «détails» dans l'ordre de la vie plus que du virtuel. D'autre part le choix de s'adresser à un collectif « vous ", « vos », " votre » crée discursivement l'entité groupe-classe, à l'intérieur duquel en toute bonne amitié on retrouve ses « petits camarades de classe ».

Pour tenter de spécifier un peu plus précisément les traits constitutifs de la relation socio-affective qui transparait dans les contributions des tuteurs, nous en retiendrons trois aspects : le soutien psychologique, la confidence, l'humour.

- Soutien psychologique 
En réponse à un message d'étudiant «j'essaie de vous envoyer ce travail par mail depuis 48 heures mais ça ne passe pas... », voici la réaction du tuteur de linguistique textuelle :

Restons «Zen » en Suisse. Même dans les montagnes, même en hiver, même à côté des vaches « tintinabullantes », on y arrive et y arrivera aussi le père Noël qui dans sa grande générosité et puisque vous avez été sage, vous apportera, vous apportera, dis-je, un...un... nordinateur. J'ai bien compris votre détresse « informatique », mais on s'en remet (moi qui vous parle je viens de perdre il y a moins de 10 minutes, toutes les données concernant le présent message !).

Les difficultés techniques sont souvent à l'origine de manifestations de frustration, de plainte voire de découragement. Pour soutenir le moral des troupes, l'humour est une arme efficace de même que les marques d'empathie telle celle qui figure en fin de message : le tuteur peut aisément se projeter dans la situation de l'étudiant puisque lui aussi peut être en proie à des problèmes du même ordre.

- Aspect confidence, implication personnelle du tuteur :

Approches interculturelles

Je relis votre contribution après avoir lu la discussion qu'elle a suscitée avec A et je repense à la discussion « animée » que j'ai eue, il y a quelques années, moi qui ne suis jamais allée au Japon avec une amie qui elle, y a vécu 7 ou 8 ans à propos du livre Stupeurs et tremblements...

Linguistique textuelle

Digression conclusive. Quand j'étais jeune et que je faisais l'imbécile sur les planches, j'ai tenu le bistrot «Chez Pierrot ». Et ça fonctionnait déjà et bien avant Gourio, sur le principe des brèves. PS : ma fille m’a demandé de ne pas en rajouter.

La participation du tuteur à la conversation se manifeste dans le premier exemple par le biais du phénomène d'échoïsation (Cosnier, 1994) que produit une discussion sur le Japon entre deux étudiants ( $c f$. corpus plus bas). Le désir de marquer son empathie se traduit par l'irruption de personnages tirés de la vie privée dans sa réponse et donc d'une certaine rupture des frontières entre le registre professionnel auquel appartient l'espace discursif sur Canufle et le registre privé auquel fait référence le tuteur. Le deuxième exemple se situe également sur le ton de la confidence dans la "digression » que le tuteur s'autorise, il est également fait référence au passé, aux souvenirs, aptes à conférer une épaisseur affective aux propos tenus aux étudiants. On soulignera ce que nous pouvons appeler « l'effet de présence » créé par le post-scriptum : tout se passe comme si la réponse du tuteur renvoyait en miroir aux étudiants le cadre familial au sein duquel elle s'élabore puisqu'on imagine sa fille commenter les lignes qu'il est en train d'écrire.

- Humour, établissement d'une connivence :

L'exemple suivant, tiré du cours " Grammaire de l'oral », comporte trois tours de parole qui constituent un échange entre l'étudiante $\mathrm{C}$ et le tuteur $\mathrm{P}$. L'étudiante va confondre $\mathrm{P}$ avec le tuteur R d'un autre cours (effet de virtuel ?) :

Etudiante $\mathrm{C}$ le $7 / 01:$ «Monsieur $\mathrm{R}$, je vous demande d'être patient, $\mathrm{j}$ 'avance à petits pas : après des problèmes d'ordinateur avec mon Mac en novembre-décembre (réglé grâce au Père Noël...Les enfants ont raison : « il est trop cool le Père Noël »), j'ai récupéré le document 5 , fort utile pour la transcription, il y a peu (soupir). Je continuerai (re-soupir) et vais tâcher de vous envoyer la transcription aussi vite que possible (re-re-soupir). Merci de votre compréhension »

Même étudiante le $8 / 01$ : «Oh, pardon, vraiment désolée, voilà dans quel état ça me met la transcription à haute dose...(N [autre étudiante] m'a signalé par mail que non seulement j'écrivais des âneries, mais en plus je me trompais de nom de prof'...) Accepterez-vous, Monsieur P, mes excuses?

Tuteur $\mathrm{P}$ le 9/01 : «C $\mathrm{C}$ après consultation avec moi-même, je et moi avons décidé de vous absoudre : transcrivez en paix mon enfant et n'oubliez pas de faire des exercices de respiration pour retrouver toute votre sérénité. Personnellement je ne souffre pas de confusion avec mon collègue R. J'espère simplement que la réciproque est vraie...Allez, bon courage, nous avons tous hâte de lire cette transcription qui vous met dans un tel état ! Cordialement.

On retiendra de cet échange tout d'abord l'aspect ludique qui fait jouer l'étudiante sur les "soupirs" communs à la convention de transcription (renvoyant à l'exercice demandé) et aux marques de lassitude répétées (« re », « re-re» soupirs) qu'elle ressent du fait de la gêne technique subie. Ensuite, l'importation dans ses propos de discours sociaux qui ne figurent pas habituellement dans des interactions écrites entre enseignant et étudiant ( « il est trop cool le Père Noël ») et qui sont la marque d'une confiance dans le degré d'intimité autorisé par la communication en ligne avec ce tuteur. Enfin, la réponse du tuteur qui rebondissant sur les soupirs propres à essouffler l'étudiante lui suggère des exercices de respiration en même temps qu'il renvoie son amusement à propos de la méprise patronymique par un jeu (je) de dédoublement. De toute évidence, la communication entre ces deux acteurs est détendue et propre à favoriser l'apaisement des tensions. 
Nous nous sommes jusqu'ici centrés sur les discours des tuteurs et avons mis en évidence différentes stratégies discursives propres à mettre à l'aise les étudiants, à faciliter la communication entre les différents acteurs de la formation en insufflant une certaine liberté d'expression. Le type d'activité proposé, impliquant personnellement les étudiants, nous semble induire une conséquence intéressante sur le plan des relations socioaffectives entre étudiants. En effet, au-delà du contenu strictement pédagogique, les étudiants qui lisent les contributions de leurs collègues ont par là même accès à certains aspects de leur vie personnelle, ils vont connaître leur façon de résoudre tel problème, de répondre à telle question en fonction de leur univers de référence. Ainsi se transmettent des éléments d'information "extra-pédagogiques" qui, dans les cours traditionnels, se diffusent hors classe, dans les couloirs ou au hasard des rencontres. Ici, le «tout en ligne collectif » implique qu'une partie de la vie privée des acteurs (celle qu'ils choisissent d'intégrer dans leurs productions) est rendue publique (à la différence des copies qui conservent l'unidirectionnalité de la communication entre l'étudiant et le professeur) et que donc, contrairement à ce qui se passe dans la classe en présentiel, tous les acteurs savent tout ce qui est dit par et sur les autres. L'analyse va maintenant se terminer par les situations d'interaction entre étudiants.

\section{Les situations d' interaction entre étudiants}

On distinguera diverses situations significatives des types d'échanges susceptibles de se produire entre étudiants. Nous nous intéresserons principalement aux interactions visibles sur la plate-forme, mais il faut signaler que d'autres échanges ont lieu entre étudiants, hors plate-forme Canufle. Ainsi, on a vu précédemment qu'une étudiante avait été prévenue par une autre, par courrier électronique, du fait qu'elle intervertissait les noms de tuteurs. On peut également signaler le fait que des étudiants ont été amenés à se rencontrer en face à face pour se dépanner : « Après bientôt 3 semaines, je sors de ma grotte, I (autre étudiante) n'aura plus besoin de me prêter son ordinateur et de faire retentir le tam-tam du Canufle dans les plaines et entre les montagnes suisses. » (linguistique textuelle, 9/12).

Nous distinguerons deux aspects : d'une part, les marques de ce que nous appellerons l'interaction cognitive, c'est à dire le fait que les étudiants s'appuient sur les contributions des autres afin de répondre eux-mêmes aux questions posées. D'autre part, les interactions communicatives, c'est-à-dire la succession, l'emboîtement des contributions étudiantes sur la plate-forme. Le premier aspect peut être illustré par deux exemples :

Approches interculturelles et didactique des langues

Etudiant A le 13/12 : Je viens juste de lire l'excellente contribution de S [autre étudiante]. Il me semble que son analyse est très détaillée et très claire.

Production de matériel didactique : la séquence pédagogique

Etudiante I le 4/12: J'ai lu avec profit les participations des autres étudiants dont je cite des extraits (« Tous les élèves d'une même classe auront une adéquation différente aux stratégies du professeur (comme le dit très bien $\mathrm{S}$ ) [...] Une méthode purement audio-visuelle [...] semble présupposer, je cite A, que tout ce qui est enseigné est acquis

Dans le premier exemple, la modalisation appréciative portée sur la production d'un autre étudiant marque un lien entre sa façon de répondre au problème posé et celle de l'autre étudiante; de façon encore plus nette, le second étudiant reprend sous forme de citations dans sa contribution les termes mêmes employés par d'autres étudiants. On voit ici de quelle façon s'effectue l'apprentissage, à travers l'appropriation des idées émises précédemment par d'autres.

Pour étudier le fonctionnement des interactions communicatives dans leur articulation les unes aux autres, nous partirons de trois situations discursives dont nous chercherons à spécifier la fonction pragmatique :

- Situation 1 : elle est extraite du cours de linguistique textuelle et montre un dialogue entre une étudiante (S) et un tuteur, dialogue dans lequel vient s'insérer une autre étudiante $(\mathrm{N})$ :

6/12 Activité décembre, étudiante $\mathrm{S}$

(re)bonjour. L'activité 2 est en attaché.

13/12 Trop simple (réponse du tuteur)

Trop simple. En effet, si l'on se place...

01/01 Réf. : Trop simple (étudiante S)

Vous trouverez ci-joint des éléments de réponse à vos questions et remarques [...]

08/01 Réf. : Trop simple (tuteur)

Votre contribution montre que vous avez compris la nature de la difficulté, et c'est un premier pas vers la résolution du problème. Je vais essayer d'expliciter cette difficulté « résistante[....].

11/01 Réf. : Trop simple, étudiante S 
Désolée d'insister mais « ça » résiste encore !... Je crois avoir compris [....] mais je décroche à partir de ......

12/01 Réf. : Trop simple, étudiante N

Bonjour $\mathrm{S}$, ce n'est pas la réponse que tu attendais, mais j'ai pensé qu'on pourrait tenter de se dépatouiller de cette activité ensemble. Après les explications de $\mathrm{Mr} \mathrm{X}$ (tuteur), j'ai eu l'impression de comprendre de quoi il retournait, mais ce n'est peut-être qu'une impression. Bref, regarde, si tu veux bien, le fichier attaché et dis-moi si ça éclaire ta lanterne ou si je suis tombée à côté ! N

13/01 Réf. : Trop simple, étudiante $\mathrm{S}$

Merci N d'éclairer ma lanterne !... J'aime bien ton expression «passage obligé » qui me semble bien expliciter ce dont il était question dans l'explication de Mr (nom du tuteur) [...]. Merci surtout de ta disponibilité pour ces échanges qui donnent une valeur ajoutée à cette formation, disponibilité dont $\mathrm{j}$ 'ai personnellement bien du mal à faire preuve [...] Cordialement $\mathrm{S}$

13/01 Réf. : Trop simple, étudiante N

$\mathrm{S}$, merci pour ta réponse, $[\ldots]$ cordialement $\mathrm{N}$

L'intervention d'une tierce personne dans le dialogue entamé s'inscrit dans un mouvement d'entraide, de soutien apporté à une camarade en difficulté. Dès le départ, l'usage du tutoiement crée une sensation de proximité entre les deux étudiantes de même que l'emploi du pronom indéfini « on » qui associe les deux étudiantes dans une même résolution de problème (« on pourrait tenter de se dépatouiller de cette activité ensemble »). Par empathie pour sa collègue, $\mathrm{N}$ vient en quelque sorte à son secours en lui offrant ses propres reformulations des commentaires du tuteur en fonction de ce qu'elle pense être les points de résistance à leur compréhension. En terme d'apprentissage, on retrouve alors le travail effectué sur la zone proximale de développement qui peut être aussi accessible aux pairs qu'aux enseignants, les premiers ayant en outre l'avantage de partager un code langagier facilitant l'intercompréhension.

- Situation 2 : dans le cours « Lettres et arts contemporains", une étudiante (V), partant de la lecture qu'elle a faite d'un poème proposé par une autre étudiante $(\mathrm{N})$, lui signale une autre rime qui aurait pu « marcher » dans le poème :

N., pour la rime pas frime, $12 / 01$

$\mathrm{N}$, « ont pu boire » marchait aussi ....Amicalement, V.

Réf. : N., pour la rime pas frime $13 / 01$

$\mathrm{V}$,

C'est promis,

$\mathrm{Si} \mathrm{je} \mathrm{sèche}$

De nouveau

Sur l'exo

Comme pimbêche

Je ne suis

Je t'écris !

Au risque de me répéter : ça fait plaisir de te savoir (enfin) connectée.

Réf. : N., pour la rime pas frime 14/01 (tuteur)

Les réactions en chaine sont, dans certains domaines, particulièrement bienvenues !

A la prochaine..

Ici, nous nous situons moins dans une démarche d'entraide (puisque, somme toute, le poème fonctionnait déjà sans cette suggestion) que dans une perspective communicative de l'ordre du phatique (pour V) et du remerciement ludique (pour N). D'une part, le ton est donné autant par le titre «pour la rime pas frime » que par la clôture "amicalement» et en retour il attire un jeu autour de la forme poétique qui, par une sorte d'acquiescement tacite, accentue le mouvement sur le travail poétique initié par la première contribution, en « rajoutant » encore plus de poésie... On a ici également un exemple de la liberté de ton qui s'est instaurée et qui peut, comme on le voit, aller assez loin dans le jeu et la complicité même si c'est « à découvert ».

- Situation 3: Deux étudiants $\mathrm{B}$ et $\mathrm{A}$, tous deux en poste au Japon, entament un échange à propos des représentations qu'ils ont de ce pays, dans le cours « Approches interculturelles ». Interviennent ensuite le tuteur et une autre étudiante $(\mathrm{N})$ :

TD de novembre / B, 24/11

Incompréhension/malentendu dus au non-dits : Les Japonais n'aiment pas dire non, ils utilisent des formes détournées (souvent comportementales) qui pour l'interlocuteur averti signifient le refus mais qui sont souvent la cause de malentendus...

Impressions japonaises, A, 26/11

Cher collègue, Je suis moi-même au Japon depuis 4 ans maintenant où j'enseigne à l'université des langues étrangères de Nagasaki. En lisant votre contribution de ce mois-ci je me suis rendu compte à quel point le débat sur 
l'interculturel est un débat personnel. En effet, même si nous habitons tous deux au Japon, je ne partage pas certaines de vos opinions. Les images que nous nous faisons de certaines coutumes sont diamétralement opposées. [...] $\mathrm{J}$ 'attends avec impatience votre prochaine réponse et $\mathrm{j}$ 'espère que nous aurons l'occasion de se rencontrer prochainement

Réf. : TD de novembre / B, tuteur, 27/11

Je relis votre contribution après avoir lu la discussion qu'elle a suscitée avec $\mathrm{A}$ et je repense à une discussion « animée » que j'ai eue il y a quelques années, moi qui ne suis jamais allée au Japon, avec une amie....

Article sur la gestion du sommeil en France et au Japon, N, 2/12

J'ai lu avec intérêt l'échange précédent. Il se trouve que je suis tombée, l'an passé, sur un article intitulé La conceptualisation de la culture : l'exemple de la gestion du sommeil en France et au Japon (lien vers ce texte) qui m'a paru très intéressant et sympathique [...] J'aimerais avoir votre opinion à tous deux sur les données qui sont présentées dans l'article, bien sûr, si vous trouvez le temps pour cette lecture. Cordialement

A dormir en France et au Japon, 3/12

Merci $\mathrm{N}$ d'avoir porté à notre connaissance cet article. J'ai profité de ma pause déjeuner pour le lire dans ses grandes lignes. Tout d'abord, je dois dire que je connais bien les auteurs de cet article [...] Pour ma part je n'adhère pas à ce mouvement (faire parler les étudiants dés les premières leçons) dans la mesure où je suis un adepte de Krashen et de sa « silent period ». Pour revenir au sujet qui nous intéresse, je suis d'accord avec certaines de leurs conclusions.

N- Réponse à A, 10/12

Bonjour $\mathrm{A}$, désolée $\mathrm{j}$ 'ai mis beaucoup de temps à vous répondre. Je suis actuellement à Fès pour une recherche de "terrain", ce qui modifie la fréquence de mes connexions aux $\mathrm{Td}$. Vos remarques m'ont permis de modifier la lecture que j'avais de cet article. Effectivement..[...] A la vérité, j'avais préparé une réponse à... votre réponse où je revenais sur l'usage de silent period, qui m'intéresse. [...] J'ai l'impression d'avoir eu recours à une sorte de silent way dans mon apprentissage de l'arabe marocain (par immersion). Je pars de mon expérience d'apprenante [...] Je me demandais si vous aviez vousmême testé, du côté apprenant cette foi, cette façon d'apprendre peut-être pour le japonais. Voilà pour l'instant. Bien cordialement. N

Si l'on cherche à préciser la fonction pragmatique de la première intervention de $\mathrm{N}$, on peut dire qu'il s'agit d'un apport d'information allant dans le sens de la conversation à bâtons rompus qui est en voie de s'instaurer entre les participants au forum : elle indique un article, proposé en lien hypertextuel, qui vient apporter un élément de plus à verser au dossier qui a été ouvert. En même temps, elle stimule l'échange déjà engagé en demandant explicitement des retours aux deux étudiants vivant au Japon ( $« \mathrm{j}$ 'aimerais avoir votre opinion à tous deux »). L'échange initié, donc, sur le mode du polylogue va déboucher sur un dialogue entre $\mathrm{A}$ et $\mathrm{N}$ qui met en évidence un début de personnalisation de la relation dans la mesure où elle débouche sur des préoccupations, une curiosité d'ordre personnel plutôt que directement liée au cours. On se situe ainsi dans une relation interpersonnelle « je me demandais si vous aviez testé... » du type de celles que l'on peut avoir, hors classe, avec un autre étudiant (il est probable d'ailleurs que la suite de la conversation ait eu lieu en dehors du forum, sauf à penser que la demande soit restée sans réponse).

Le lecteur attentif aura remarqué que la même étudiante $(\mathrm{N})$ est impliquée dans les trois exemples d'interactions entre étudiants. C'est elle qui fait irruption dans un débat pour ajouter une information (situation 3) ou pour aider une autre étudiante (situation 1). En retour c'est à elle que l'on signale une autre version possible de sa production poétique (situation 2). D'où certaines questions que l'on peut se poser : certains individus seraient-ils plus spontanément disposés à interagir avec d'autres? Ne s'agit-il pas d'une forme d'autonomie dans les apprentissages à distance que cette capacité à entrer en relation avec ses pairs tant sur le plan socio-affectif (qui permet de lutter contre le sentiment d'isolement) que sur le plan socio-cognitif (qui permet une certaine construction sociale des connaissances) ? Enfin, si le rôle du tuteur semble moins central dans ce type d'interactions entre pairs, celles-ci existeraient-elles sans lui et notamment sans des tâches incitant à différentes formes de coopération ou de mutualisation?

\section{Conclusion générale}

Le corpus retenu pour cette étude est un peu réduit en termes temporels (deux mois) pour que l'on puisse prétendre avoir fait une analyse exhaustive de la qualité du lien social en construction au fil des échanges qui se sont effectués en ligne entre étudiants. Néanmoins on a pu montrer qu'à différents niveaux s'exprimaient des marques de convivialité et de soutien entre pairs. Le passage au tutoiement, aux salutations affectivisées ( $c f$. « amicalement ») vont de pair avec la mise en place d'un sentiment d'appartenance à un collectif, Canufle, qui s'exprime très explicitement dans les vœux de bonne année d'une étudiante le 30/12/03 :

Meilleurs vœux à vous et à tous mes collègues canufliens et canufliennes! (I, cours Grammaire de l'oral) 
Ces désignations, assorties de variations (on a vu «canuflistes» plus tard dans l'année), paraissent représentatives du désir d'inscrire en termes identitaires l'appartenance des étudiants à cette formation en ligne. Tout se passe comme si, du fait qu'ils sont dispersés dans le monde, les étudiants cherchaient à « faire corps », à constituer une entité repérable entre eux, en toute «intimité virtuelle». On peut admettre que la fluidité des échanges qu'autorisent les forums en ligne entre pour une part non négligeable dans la création de ce lien social. On peut également penser que le dévoilement de soi lié au fait de communiquer quasiment en direct mais sans être vu est un élément désinhibant qui mériterait d'être étudié plus spécialement pour le rôle qu'il peut jouer dans les contextes éducatifs.

L'analyse des discours en ligne dans ce campus numérique ne visant pas la collaboration nous a donc permis de repérer un certain nombre d'opérations langagières permettant d'avancer d'une part qu'un certain « sentiment de communauté » se fait jour dès le début de la formation grâce notamment - mais pas uniquement - aux incitations, aux tâches et aux feed-back des tuteurs, d'autre part que ce sentiment comporte deux dimensions également importantes et souvent étroitement imbriquées, le socio-affectif et le socio-cognitif. On suggèrera pour conclure que l'analyse du discours appliquée à des formations en ligne ayant déjà eu lieu, outre son intérêt en tant que méthodologie de recherche, pourrait constituer un intéressant outil de formation des tuteurs. Cette suggestion ne serait sans doute pas contredite par Lamy (2001: 142-143), qui insiste sur les difficultés inhérentes à la dimension purement verbale de l'interaction en ligne :

La gestion de l'interaction en ligne est d'autant plus problématique que les participants n'ont aucun contact présentiel. Dans ce cas [...] il existe un déficit socio-affectif au départ, qu'il est important de compenser. Or le seul « lieu» où puisse intervenir ce ré-équilibrage est un lieu purement verbal, et n'est en fait qu'une élaboration textuelle collective. Le pédagogue en ligne doit donc se construire par l'unique biais du discours un rôle nouveau, prenant en compte les propriétés matérielles de l'outil (interface, utilisation de l'espace et du temps) ainsi que les " comportements » autant cognitifs que socio-affectifs d'une communauté d'apprentissage créée elle aussi de toutes pièces par un ensemble de discours.

\section{Bibliographie}

Arnaud M. (2003) «Les limites actuelles de l'apprentissage collaboratif en ligne», Revue STICEF, Volume 10, 2003, Rubrique, mis en ligne le 15-11-2003, http://sticef.org

Averous M. et Touzot G. (2002) «Campus numériques, enjeux et perspectives pour la formation ouverte et à distance. Rapport établi en avril 2002. Consulté en juin 2004 : http://www.educnet.education.fr/superieur/rapports.htm

Bonamy J. \& Charlier B. (2003) “Un dispositif efficace?”, in Charlier \& Peraya, p. 181-195.

Brown R. E. (2001) "The Process of Community-building in distance learning classes", in Journal of Asynchronous Learning Networks, Vol. 5, Issue 2. Revue en ligne: http://www.aln.org/publications/jaln/

Charaudeau P., Maingueneau D. (dir., 2002) Dictionnaire d'analyse du discours, Paris, éditions du Seuil.

Charlier B., Peraya D. (éds., 2003) Technologies et innovation en pédagogie. Bruxelles, De Boeck Université.

Castells M. (2001) La galaxie Internet. Paris, Fayard.

Conrad D. (2002) "Deep in the Hearts of Learners: Insights into the Nature of Online Community", in Journal of Distance Education / Revue de l'éducation à distance.

Cosnier J (1994) Psychologie des émotions et des sentiments, Paris, Retz Nathan

Daele A., Charlier B. (coord., 2002) Les communautés délocalisées d'enseignants. Etude réalisée dans le cadre du Programme numérisation pour l'enseignement et la recherche. Disponible sur Internet (juin 2004) :

Denis B. (2003) « Quels rôles et quelle formation pour les tuteurs intervenant dans des dispositifs de formation à distance ?», Distance et savoirs Vol. 1, num. 1, p. 19-46.

Deschryver N. (2003) « Le rôle du tutorat », in Charlier \& Peraya (éds.), p. 149-162.

Develotte C. (2004) «L'étudiant en autonomie et ses professeurs virtuels : comment se personnalise la relation en ligne?», communication au colloque UNTELE 2004, cédérom édité et diffusé par l'université de technologie de Compiègne.

Dillenbourg P., Poirier C., Carle L. (2003) «Communautés virtuelles d'apprentissage : e-jargon ou nouveau paradigme ? », in Taurisson \& Sentini (dir.), p.11-47.

Flichy P. (2001) L'imaginaire d'Internet. Paris, La Découverte.

George S. et Leroux P. (2003) «Un environnement informatique de soutien à une pédagogie de projet: un contexte d'apprentissage à distance en robotique pédagogique », in Taurisson \& Sentini (éds.), p. 73-102. 
Henri F., Lundgren-Cayrol K. (2001) Apprentissage collaboratif à distance. Presses de l'université du Québec.

Henri F., Pudelko B. (2002) « La recherche sur la communication asynchrone : de l'outil aux communautés », in Daele, A. \& Charlier, B. (coord.), p. 12-44.

Kerbrat-Orecchioni C. (1997) L'énonciation, Paris, Armand Colin/Masson.

Lamy M.-N. (2001) «L'étude d'une langue vivante assistée par ordinateur: réflexion collaborative sur l'objet d'apprentissage ", in Bouchard R. et Mangenot F. (éds.) Interactivité, interactions et multimédia, Notions en Questions $\mathrm{n}^{\circ} 5$, p.131-144, Lyon, ENS-Editions.

Mangenot F. (1999) Technologies de l'information et de la communication et enseignement-apprentissage du FLE. Cours de maîtrise de français langue étrangère. Poitiers, CNED.

Mangenot F. (2002) «Forums et formation à distance : une étude de cas ». In Education permanente 152. pp. 109-119.

Mangenot F. (2003) "Tâches et coopération dans deux dispositifs universitaires de formation à distance », in $A L S I C$ (Apprentissage des langues et systèmes d'information et de communication) Vol. 6, num. 1, p. 109-125. Revue en ligne : http://www.alsic.org

Oliver D., Herrington J. (2001). Teaching and Learning Online. Perth : Edith Cowan University.

O'Regan K. (2003) "Emotion and E-Learning”, Journal of Asynchronous Learning Networks, Vol. 7, issue 3. Revue en ligne: http://www.aln.org/publications/jaln/

Taurisson A. \& Sentini, A. (dir., 2003) Pédagogies.net. L'essor des communautés virtuelles d'apprentissage. Presses de l'Université du Québec.

Turkle S. (1997), Life on the Screen, Identity in the Age of the Internet, Touchstone Book, Simon \& Schuster, New York, 1997 ( $1^{\text {ère }}$ éd. 1995). 